\title{
Malignant external otitis in diabetic patients
}

\begin{abstract}
Malignant external otitis (MEO) or necrotizing otitis, is an infectious pathology rare but serious to the external ear canal (EEC), predisposed field and diabetes, she finds the ideal conditions for its development in this field and exposed to the risk of bacterial superinfection. It is a descriptive study of 12 patients with diabetes seen in the emergency room of the university hospital Mohamed VI Marrakesh, Morocco. All our patients had an MEO manifested by a discreet, banal, earache that gradually will intensify, be accompanied by a variable but typically festering abundance otorrhoea. The average age of patients was 70 years. Seniority of diabetes was 9 years at $90 \%$. It is essentially type 2 diabetes imbalanced $(70.5 \%)$. There is a fickle hearing loss and moderate at $35.3 \%$. Facial paralysis full at $58.8 \%$ of the patients. The more found germ is Pseudomonas aeruginosa (90\%). Antibiotic therapy adapted to the antibiogram is prescribed, with intensified insulin therapy. The predisposed field is essentially diabetes ( 75 to $95 \%$ of the cases in large series of literature). The occurrence or severity of MEO is not correlated to the imbalance of diabetes, however this imbalance. Bacteriological examination and the antibiogram and medical imaging should be systematic, after confirming diagnosis, adequate treatment avoiding the basicranienne extension. This became almost exclusively medical with the appearance of antipyocyaniques antibiotics which have transformed the once formidable prognosis. Stop antibiotic therapy is conditioned by the standardization of the scan to the Galliu, an optimized insulin therapy is fundamental. The overall mortality is less than 20 percent today. Malignant external otitis or external otitis progressive Necrotizing is a serious infection of the subject the often older and diabetic by Pseudomonas aeruginosa. There are prevention by avoiding all the traumatic maneuvers of the EEC, and especially think about in order to establish as early as possible after confirmation diagnostic.
\end{abstract}

Keywords: diabetes, malignant external otitis, supported
Volume 6 Issue 3 - 2018

\section{Bahia Habra, Ghizlane El Mghari, Nawal El Ansari}

Service of Endocrinology, diabetes and metabolic diseases, the university hospital Mohamed VI. Faculty of medicine and pharmacy, University Cadi Ayad, of Marrakesh, Morocco

Correspondence: Bahia Habra, Service of Endocrinology, diabetes and metabolic diseases, the university hospital Mohamed VI. Faculty of medicine and pharmacy, University Cadi Ayad, of Marrakesh, Morocco, Tel 212672520912 , Email b.habra2013@gmail.com

Received: February 14, 2018| Published: May 07, 2018

\section{Introduction}

Malignant external otitis (MEO) or necrotising otitis is a rare entity, the starting point is a violation of the external ear canal (EEA) and dissemination of the infection to the soft parts and the adjacent bone, hard adiagnostiquer and whose evolution can be very serious. Elective on debilite ground, such as immunocompromised, or more often the elderly diabetic, is a necessary diagnostic criterion. The symptoms and signs may include severe earache, otorrhoea and achievement of various crannies, often caused by Pseudomonas Aeruginosamais also of other agents can rarementinclure to other bacteria (Staphylococcus aureus, Proteusmirabilis, Klebsiellaoxytoca, Pseudomonascepacia) or fungi (Aspergillus, Pseudallescheria, Candida, Pityrosporum). ${ }^{1}$ The presence of tissue granulation in the EEC, a high rate of sedimentation of red, and abnormalities on imaging studies are also diagnosis parameters. It is so very important to make the diagnosis of MEO as soon as possible, at the beginning it is very similar to severe acute external otitis, but MEO is developing a severe osteomyelitis of the temporal bone, attaquantles cranial nerves adjacent (VII, XII), the blood vessels and soft tissue, and finally, if it is not treated, it causes death due to the expansion osteomyelite of the skull and thromboembolism septic disseminated brain. Therapy should be should be conducted by the otolaryngologists in collaboration with the endocrinologist, internist, neurologist, radiologist and microbiologist. Local treatment (with removal of bone sequestered) and systemic antibiotic treatment according to the results of bacteriological tests (aminoglycosides, semisynthetic Penicillins, third- and fourthgeneration cephalosporins, fluoroquinolones) are generally accepted.

\section{Materials and methods}

Descriptive study spread over 4 years, including 12 diabetic patients with an MEO seen in our emergency room. We will study the clinical, diagnostic and progressive aspects of malignant otitis externa and management of this pathology and a literature review. Studied parameters: epidemiological characteristics of the population: age, sex, weight, size, origin, nature of work, pets. Characteristics of diabetes: type, age of onset, age of diabetes, diabetes tracking duration of evolution, presence or not of degenerative complications of diabetes, treatment, Glycemic balance assessed by $\mathrm{HbA1C}$ or the glucose cycle, Triggers, Characteristics of the lesion, Bacteriological sampling and paracliniques examinations and Therapeutic support.

\section{Results}

We saw 12 diabetic patients with an MEO, the average age 58 years of extremes between 42 and 70 years. Female: $79.1 \%$, a sex ratio $(\mathrm{F} / \mathrm{H}): 3.8$; all patients were diabetic type 2 . Average diabetes 8.9 years, extreme seniority: 2 years to 20 years. All patients were carriers of a lesion of the EEC that would reveal a patient MEO and 11 was under oral antidiabetic agents (ADO), two patients on insulin. Some triggers reported in $20 \%$ of patients with a swim in the Moorish bath, an aspiration, a foreign body, an ear scratch, Auricular trauma. The 
majority of patients were in blood glucose imbalance with an average $10.9 \% \mathrm{HbA} 1 \mathrm{c}$, a venous hyperglycemia on admission between 2.5 $\mathrm{g} / \mathrm{l}$ and $4 \mathrm{~g} / \mathrm{l}$; the majority of our patients have a degenerative status unknown, amputee patients to knee-deep. Clinical table composed of discreet, banal, earache will gradually intensify, be accompanied by a variable but typically festering abundance otorrhoea, fickle hearing loss, tinnitus associated with rotary in Dizzy a case and headache. Peripheral facial paralysis was present at the initial examination at a patient, a hyperthermia at $38,5^{\circ}-39^{\circ}$, the EEC exam, always difficult because painful, unilateral achieve all our patients, the otoscopique review is a stenosis inflammatory full and a granulation tissue at the level of the EEA in $40 \%$, Presence of Bud at $32.5 \%$, Presence of bony or cartilaginous receiver at $7.5 \%$, the eardrum not seen in $65 \%$ of the patients, the rest of the otolaryngeal examination found Inflammation peri-auriculaire at $25 \%$, Sanitizer at $15 \%$, the isolated germs to culture in 8 patients was Pseudomonas aeruginosa in $75 \%$ of cases, Staphylococcus coagulase negative, and aureus, acinetobacter, the TDM (tomodensitometry) made in 9 patients showed a filling of the EEC and mastoidiennes cells with irregularity of the bony walls of the EEC in 7 cases, a bone lysis of the mastoid process, the tympanal bones and or of the petrous apex was noted in 5 cases (figures 1 and 2). All patients were initially treated for a trivial external otitis and it's in front of resistance to the General day-to-day treatments and the appearance of complications that patients have been referred for further exploration. Broad spectrum by parenteral antibiotics (amoxicillin $3 \mathrm{~g} /$ day; ciprofloxacin or ofloxacin clavulanic acid) adapted to the antibiogram intensified insulin therapy. Local treatment is also essential, everyday with cleaning, calibration, local debridement were made in all cases and was to remove all the granulation tissue and bone ill and necrotic. A modified radical mastoidectomy was performed on the wall of the canal, a nervous decompression was done in the patient with paralysis of the facial nerve. The patients were followed for at least 6 months. A patient is still under surveillance. The patient with paralysis of the facial nerve was also examined and followed by neurologists, ophthalmologists and physiotherapists.

\section{Discussion}

The MEO is an otological infection that has life-threatening complications. It is considered as a complication of external otitis. $^{2}$ Occurs mainly in the elderly who have diabetes or another condition that compromises the immune system. Doctors must be able to recognize this infection, initiate treatment, and refer patients to an Otolaryngologist. The precise etiology of this condition is unknown, but theories related to impaired immunity, the local tissue microangiopathie and even modified biochemistry of earwax have been proposed. Infection of the soft tissues of the external ear canal is common, especially in hot and humid climates. The usual triggers are trauma (often caused by q-tips) and exposure to the pool of water. The most frequently found germ is Pseudomonas aeruginosa, is not a normal germ of the EEC. ${ }^{3}$ Other possible germs include Staphylococcus epidermidis, ${ }^{4}$ gram negative bacteria and fungi. External otitis patients complain of earache and sensitivity to atrial movement. The otorrhoea may be present, the obliteration of the external ear canal by edema and secretions can cause hearing loss, or a feeling of fullness in the ear. The disease comes from the EEC and propagates through the osteocartilaginous junction to involve soft tissue under the temporal bone. Granulation tissue is usually present in the EEC. The spread of the infection can cause thrombosis of the lateral sinuses and petrous sinus upper and lower. Initially, the progressive skull base osteomyelitis can cause cranial Polyneuropathy, paralysis of the facial nerve being the most common; cranial nerves IX, X, and XI (the jugular foramen syndrome) nerves and cranial nerve XII (hypoglosse channel) are less often involved with the development of the sphenoidal sinusitis. Imaging to show the extension of the infection to the bony structures is usually required to establish the diagnosis of $\mathrm{MEO}^{6}$ imaging modalities include tomographic (CT) scan, scan bone to the technetium Tc99m medronate methylene diphosphonate, and scintigraphy to $\mathrm{Ga}$ 67 gallium citrate. CT is used to determine the location and extent of diseased tissue (Figure $1 \&$ Figure 2). The temporal bone is the first os to be affected, with an imminent involvement of petrous apex and the mastoid process. Extratemporal extension has become rare since the introduction of powerful antibiotics. In evaluating the scanner, it is important to remember that at least one third of the minerals is lost before the radiological changes are becoming apparent; Conversely, the bone remineralization continued long after that infection is Gueriela scan is a diagnostic sensiblemais non-specific to the MEO, if to the $67 \mathrm{Ga}$ scintigraphy is available, (Figure 3 ). It must be used for the initial diagnosis and follow-up tool. ${ }^{7}$ Using in combination imaging modalities, it is possible to prove that the temporal bone is reached (scan and bone scan to the $99 \mathrm{mTc}$ ) with infectious process (Ga 67 scintigraphy). ${ }^{6} \mathrm{MRI}$ is of interest in advanced forms allowing a better analysis of the deep spaces of the face, the nervous foramina, merien hard spaces and the Medullary bone in the base of the skull.

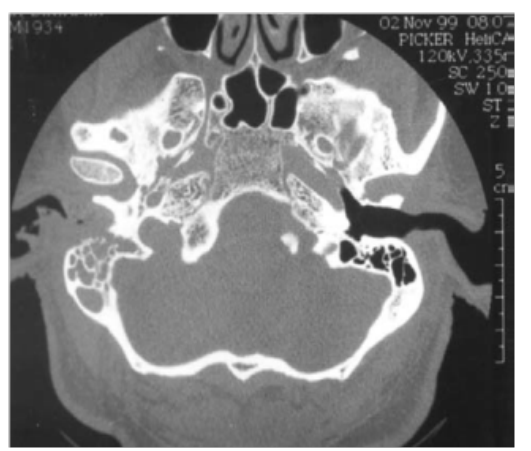

Figure I Axial scanner (TDM) in a patient aged diabetes, refereen reason for the paralysis of the facial nerve that has grown despite the oral treatment. The scanner shows the right temporal bone destruction. Note the disappearance of the posterior wall of the external ear canal (short arrow). Mastoid air cells are secondarily involved and clouded (long arrow) from the left side well ventilated.

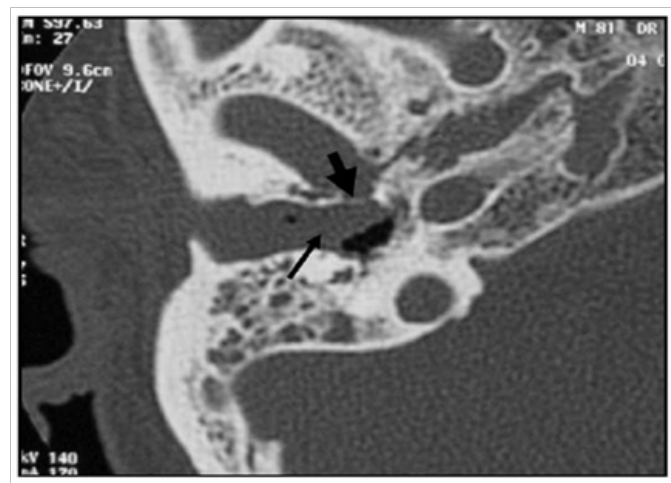

Figure 2 TDM from the rock, axial bone window: lysis of the tympanal bones. 


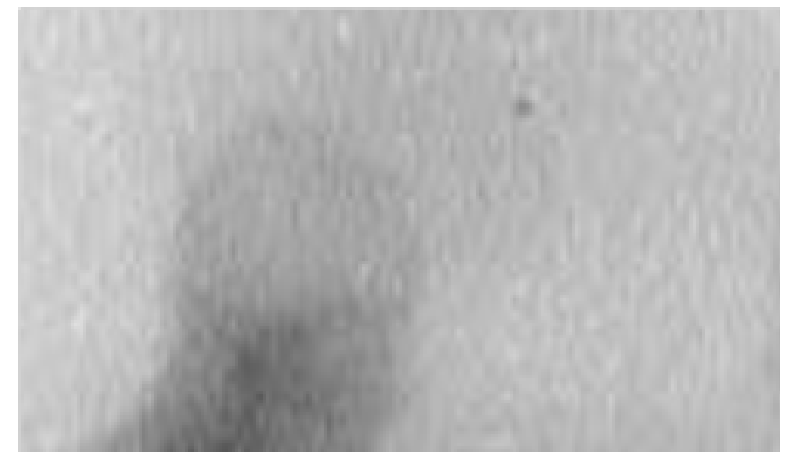

Figure 3 The left temporal bone shows an increased absorption of $67 \mathrm{Ga}$ (arrow).

The standardization of scintigraphy has a predictivede healing value. The treatment is based on antibiotic therapy extended replacing more surgical treatment. Is followed by CT to assess the decline in inflammatory lesions of the deep spaces of the face and bone necrosis. A strict control of diabetes mellitus is mandatory, although it may be difficult to achieve during the acute illness. Other immunosuppressants and comorbid States must also be managed effectively. Local treatment of the ear canal includes the cleaning and debridement more topical application of antimicrobial agents (antibiotics and other). Escrow and other necrotic tissues must be removed. Hyperbaric oxygen, an adjuvant to antibiotic therapy, supposed to increase the capacity of the cells polymorphonucleaires against pathogenic bacteria. ${ }^{8}$ However, the complexity of administration often limits the use of hyperbaric oxygen therapy. The surgery has a defined but more limited role in the treatment of the Osteitis of the base of the skull. Although the sequestered bone and abscesses are treated surgically, a new expanded operation can be counterproductive because the healthy bone may be exposed to infection. ${ }^{6}$ The differential diagnosis is done essentially with a neoplastic origin and more rarely with pseudo tumor inflammatory, Wegener's Granulomatosis. ${ }^{9}$

\section{Conclusion}

MEO is always a serious associated life-threatening disease that requires a diagnosis and emergency treatment, you need to know to speak to an external ear infection resistant to antibiotics in a diabetic. The systematic removal of the Granuloma in the EEC. The scanner confirms the diagnosis typically showing an osteolysis of the cortical bone mastoidal and tympanale. It is performed in an emergency before any suspicion of malignant external otitis. MRI remains the test of choice; Thanks to its excellent resolution to clarify the extent of the damage to soft tissue and the nervous foramina of the skull base. The role of bone scintigraphy is important in evolutionary track of the MEO. Scintigraphy with technetium 99 allows the diagnosis at an early stage and to detect a possible extension endocranienne.

\section{Conflicts of interest}

The authors state no conflict of interest

\section{Contributions of the authors}

All the authors contributed to the conduct of this work. The authors also declare having read and approved the final version of the manuscript.

\section{References}

1. Bellini C, Antonini P, Ermanni S, et al. Due Malignantotitisexterna Aspergillus niger. Scand J Infect Dis. 2003;35(4):284-288.

2. Sander R. Otitis externa: a practical guide to treatment and prevention. Am Fam Physician. 2001;63:927-936, 941-942.

3. Meltzer PE, Kelemen G. Pyocyaneous osteomyelitis of thetemporal bone, mandible and zygoma. Laryngoscope. 1959;69:1300-1316.

4. Soldati D, Mudry, Monnier P. Necrotizing otitis externa causedby Staphylococcus epidermidis. EUR Arch Otorhinolaryngol. 1999; 256(9):439-441

5. Karaman E, Yilmaz M, Ibrahimov M, et al. Malignant Otitis Externa. $J$ Craniofac Surg. 2012;23(6):1748-1751.

6. Amorosa L, Modugno GC, Pirodda A. Malignant external otitis: review and personal experience. Acta Otolaryngol Suppl. 1996;521:3-16.

7. Stokkel MP, Boot CN, van Eck Smit BL. SPECT gallium scintigraphyin malignant external otitis: initial staging and follow up. Case reports. Laryngoscope. 1996;106(3_pt_1):338-340.

8. Davis JC, Gates GA, Lerner C, et al. Adjuvant hyperbaric oxygen in malignant external otitis. Arch Otolaryngol Head Neck Surg 1992;118(1):89 -93 .

9. Hariga I, Mardassi A, Belhaj Younes F, et al. Necrotizing otitis externa: 19 boxes ' report. EUR Arch Otorhinolaryngol. 2010;267:1193 -1198. 